\title{
Deconvolution of grading curves during milling: example of wheat straw
}

\author{
Nicolas Blanc ${ }^{1, \star}$, Vincent Richefeu ${ }^{2}$, Claire Mayer $^{1}$, and Jean-Yves Delenne ${ }^{1}$ \\ ${ }^{1}$ IATE, UMR 1208 INRA - CIRAD - Montpellier Supagro - Université de Montpellier, 2 place Viala, 34060 Cedex, Montpellier, \\ France \\ ${ }^{2}$ Université Grenoble Alpes, 3SR, F-38000 Grenoble, France
}

\begin{abstract}
The evolution of grading-curves during powder milling or agglomeration processes includes a wealth of information about the mechanisms involved at the scale of particles. However, such information can hardly be retrieved from the particle size distribution (PSD). Based on a minimization technique we developed a methodology for the decomposition of grading curves as sub-PSDs. In this paper we follow their evolution with time in the specific case of the comminution of wheat straw.
\end{abstract}

\section{Introduction}

Milling is a crucial operation for many industrial applications in pharmaceutical industry, cement production, food manufacturing... The main objective of the milling is to reduce the size of the raw material and to produce a target particle size distribution (PSD). The process involves many complex mechanisms at the scale of particles and is largely influenced by the microstructure of the material, the process parameters, and the environmental conditions. Milling is particularly important for the refinery of biomass in dry conditions [1] especially for the production of building block molecules, fibers, biocomposite fillers... In this case, several types of size reduction are generally distinguished: coarse milling ( $\mathrm{cm}$ to $\mathrm{mm}$ ) which disorganize the plant material at the scale of the organs, intermediate micronization at tissue scale ( $1 \mathrm{~mm}$ to $100 \mu \mathrm{m})$, fine grinding at cells scale $(<100 \mu \mathrm{m})$ and ultrafine grinding $(<20 \mu \mathrm{m})$ which implies the fracture of plant cell walls [2]. In a grinder, all these characteristic sizes can coexist as different populations of particles and their proportion evolves with time.

In this paper, we investigate the evolution of particle size reduction of a residue from agriculture industry: wheat straw. Wheat straw has a very heterogeneous microstructure (Figure 1) involving cells embedded in different tissues. During the milling process several populations of particles are generated leading to complex multi-modal PSDs. In this context we developed a tool to decompose the PSD as the sum of sub-distribution functions. The evolution of the characteristics of these sub-distributions (which are supposed to have specific properties in terms of grindability, chemical composition, shape...) is discussed in relation to the structure of wheat straw.

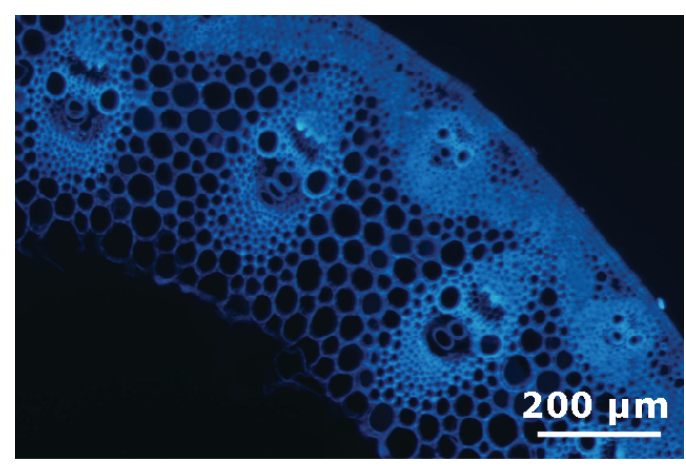

Figure 1. Cross section of an internode of wheat straw [3]

\section{Comminution of wheat straw}

We used the straw from a soft-wheat variety harvested in 2015. It was first milled in the Cutting Mill SM100 $\left(\right.$ Retsch $\left.^{\circledR}\right)$ with a selection grid of $1 \mathrm{~mm}$ aperture and sieved between 0.25 and $0.5 \mathrm{~mm}$ leading to a final median volume size $d 50=511 \mu \mathrm{m}$. This raw powder was then milled in a jar rolling mill of intern diameter $175 \mathrm{~mm}$ and volume $2 \mathrm{~L}$. The jar was filled at $33 \%$ with $3 \mathrm{~kg}$ of steel balls. The mass of the balls follow a uniform distribution per mass fraction with 3 diameters: 15,20 and $25 \mathrm{~mm}$. The mass of raw powder $(63.1 \mathrm{~g})$ was calculated in order to fill the space between the balls and to limit useless impacts between balls. The jar is rotating at $66 \mathrm{rpm}$ which corresponds to $65 \%$ of the speed of centrifugation. Samples are withdrawn all along the grinding process until a $\mathrm{d} 50$ of $7 \mu \mathrm{m}$ (milling time: $1342 \mathrm{~min}$ ) was reached.

The particle size are measured by laser diffraction using a granulometer Malvern Mastersizer 2000. Ethanol was used as dispersant and three minutes of ultrasound was applied before the measurements to maximize the dispersion of the particles. Particle sizes were determined, by

\footnotetext{
^e-mail: nicolas.blanc@supagro.inra.fr
} 
using the Mie method accurate for small particles at low concentration [4]. The size range $(0.05$ to $2000 \mu \mathrm{m})$ is divided in $N_{s}=100$ classes $i(i \in[1,100])$ following the relation:

$$
x_{i}=x_{i-1}\left(\frac{x_{N_{s}+1}}{x_{1}}\right)^{\frac{1}{N_{s}+1}}
$$

with $x_{i}$ the $i^{\text {th }}$ lower-bound of the class $i$. The PSD is built by determining the volume fraction of particle in each class. Figure 2 shows all PSDs determined during the full grinding process. The presence of the different peaks reveals the existence of several populations.

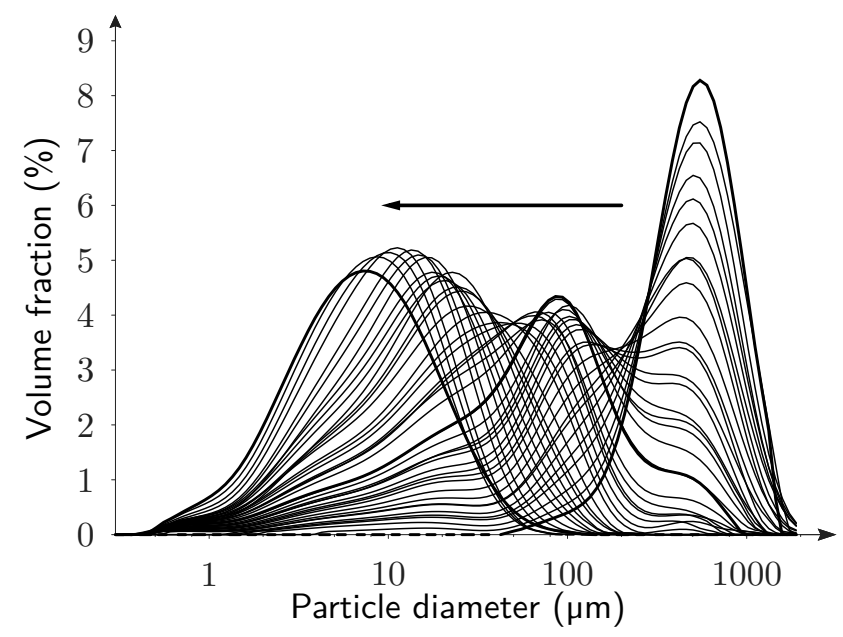

Figure 2. Evolution of PSD of wheat straw during grinding. The arrow indicates the time progression

\section{Deconvolution of grading curves}

The purpose of the methodology described below is to decompose the PSD into a sum of $N_{p}$ sub-distributions (subPSDs). We denote $N_{s}$ the number of discrete classes and the particle sizes $s^{i}$. From the shape of PSDs obtained in grinding processes it is clear that sub-PSDs have some level of asymmetry and should be bounded. For these reasons, following the works of Voivret et al. [5], we use the Beta law for each sub-distribution (i.e. for each population):

$\mathcal{V}_{p}\left(s^{i} ; \mathcal{X}_{p}\right)= \begin{cases}w_{p} \frac{\left(s_{p}^{i}\right)^{\left(\alpha_{p}-1\right)}\left(s_{p}^{i}-1\right)^{\beta_{p}}}{B\left(\alpha_{p}, \beta_{p}\right)} & \text { if } s^{i} \in\left[a_{p}, b_{p}\right] \\ 0 & \text { otherwise }\end{cases}$

where $p$ is the population of particles and $\mathcal{X}_{p}$ is a set of parameters: $w_{p}$ control the peak magnitude, $\alpha_{p}$ and $\beta_{p}$ are parameters of the normalized Beta function $B\left(\alpha_{p}, \beta_{p}\right)$, and $a_{p}$ and $b_{p}$ define the distribution range scaled to $[0,1]$ using $s_{p}^{i}=\left(s^{i}-a_{p}\right) /\left(b_{p}-a_{p}\right)$.

The grading curve, which is the master distribution, is the sum of the sub-PSD:

$$
\mathcal{V}\left(s^{i} ; \mathcal{X}\right)=\sum_{p=1}^{N_{p}} \mathcal{V}_{p}\left(s^{i} ; \mathcal{X}_{p}\right) \text { with } \mathcal{X}=\bigcup_{p=1}^{N_{p}} \mathcal{X}_{p}
$$

with

$$
\sum_{p=1}^{N_{p}} \sum_{i=1}^{N_{s}} \mathcal{V}_{p}\left(s^{i} ; \mathcal{X}_{p}\right)=100 \%
$$

The core principle of the methodology is to compute sub-PSD parameters so that the master distribution best matches the experimental PSD. The latter is defined as a set of $N_{s}$ pairs $\left\{\left(s^{i}, v^{i}\right)\right\}$, where $s^{i}$ is the mean size of the class and $v^{i}$ its volume fractions.

The set $\mathcal{X}$ contains thus $N=5 \times N_{p}$ values $x_{n}$ to be optimized. To do so, a classical minimization of the mean squared of $\sum_{i} r^{2}\left(s^{i}\right)$ is achieved, where

$$
r\left(s^{i}\right)=v^{i}-\mathcal{V}\left(s^{i} ; \mathcal{X}\right)
$$

This minimization was performed thanks to the Levenberg-Marquardt algorithm [6, 7] implemented in the minpack library [8]. This iterative algorithm is known for its robustness as it is accurate even if its initial conditions are chosen far from the final solution. Note that, it is also possible to constrain the possible values of $\mathcal{X}$ by weighting the Jacobian terms $\partial r / \partial x_{n}$. For experimental reasons it is reasonable to assume that $w_{p}>0$, and $\alpha_{p}>1$ and $\beta_{p}>1$ ensuring that $\mathcal{V}_{p}$ curve is bell-shaped.

Figure 2 shows the experimental PSDs measured by laser diffraction for 41 milling times of the straw. For each PSD, we observe a maximum number of four peaks. The deconvolution of the PSD at initial time is performed by positioning approximately the sub-PSDs on the 4 peaks. The algorithm is then run to determine the best fit. For the other PSDs we use the previous sub-PSDs as initial condition. It is worth noting that the initial guess may have an influence as several solutions may exist. This procedure requires an important number of experimental PSDs in order to capture a continuous evolution of the sub-PSDs parameters. Figure 3 shows an example of deconvolution of a grading curve into 4 sub-PSDs after 5 hours of milling. Some sub-PSDs, like the one of the population 4, may have very low weight. It is worth noting that this could leads to fluctuations, for example in the determination of the position of the pic as a function of time.

\section{Analysis}

Figure 4(a) shows the evolution of the volume fraction as a function of the mode of the distribution, which corresponds to diameter at the peak. It is interesting to note that the peak diameters can be related to the characteristic scales generally observed in the grinding of plants: tissue scale (peaks 1 between 450 and $550 \mu \mathrm{m}$ and 2 around $100 \mu \mathrm{m}$ ), cell scale (peak 3 between 10 and $40 \mu \mathrm{m}$ ) and cell wall scale (peak 4 around $1 \mu \mathrm{m}$ ) (see section 1 ).

During the grinding process, the largest particles (population 1) are broken into smaller sizes. The population 2 seems to act as a transition size. The volume fraction of the population 4 remains low even for a long grinding time (more than 20 hours) showing that this size is hardly reachable with our grinding process. The right-shifting in diameter of population 3 at the beginning of the milling may be 


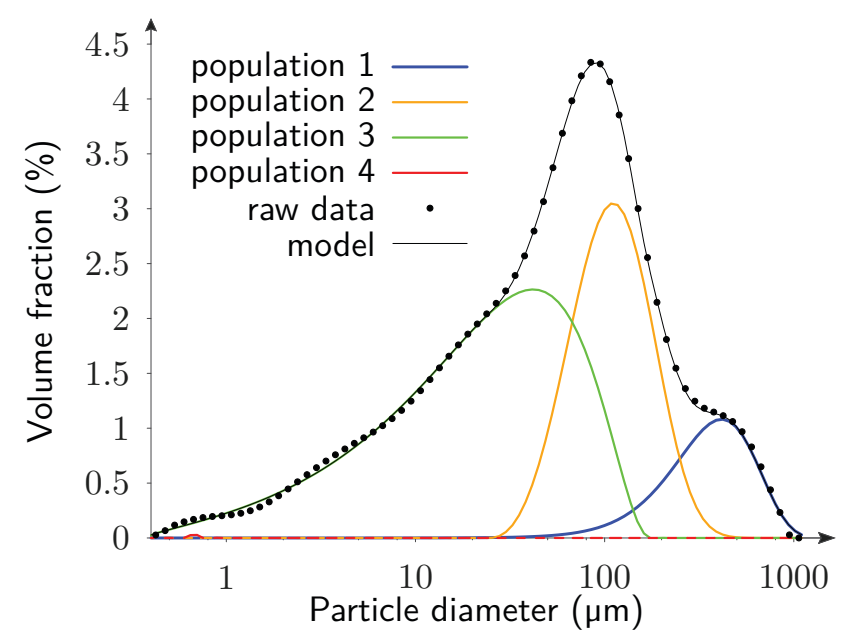

Figure 3. Example of deconvolution of the experimental PSD at a milling time of 4.78 hours.

attributed to deconvolution artifacts for weakly weighted sub-PSDs.

From Figure 3, one might think that the sub-PSD modes should decrease continuously with time as for the experimental PSD. Interestingly, the modes of population 1, 4 and partially 2 are nearly constant in the course of time; Figure 4(b). This can be due to a "shattering" process in which the particles are broken into multiple fragments feeding a limited number of smaller population with given sizes. On the contrary, the slow decrease of the mode of populations 2 and 3 probably denotes an erosion process [9] leading to a slow decrease in size of particles.

Figure 5 shows the evolution of the volume fraction with time. Four phases can be distinguished: I) (up to 4 hours) the volume fraction of populations 1, 2 and 3 evolve linearly. Population 1 decreases whereas both populations 2 and 3 increase with the same rate. II) From 4 to 7 hours, the population 2 decreases while populations 1 and 3 keep the same trend. III) Population 1 totally disappeared after 7 hours of grinding. Slopes of populations 2 and 3 change, corresponding to the time when their modes start to decrease. This indicates a possible erosion process and seems to be consistent with the increase in volume of finest particles (population 4). At the beginning of the process the stress is probably transmitted though the biggest particles while the smallest ones, into the pores network, are globally protected [10]. During this phase, populations 2 and 3 weakly evolve. Possible reasons are that erosion is less efficient than shattering, the remaining particles are the strongest or the increase in specific surface leads to a greater dissipation in friction and collisions. During this phase, the population 3 represents nearly the totality of the product (90\% at 7 hours). Finally, in phase $I V$ ), after 12 hours, only populations 3 and 4 remain.

The mechanical energy transmitted to particles is directly proportional to milling time. Thus the variation rate of volume fraction depends on the milling ability of a given population.
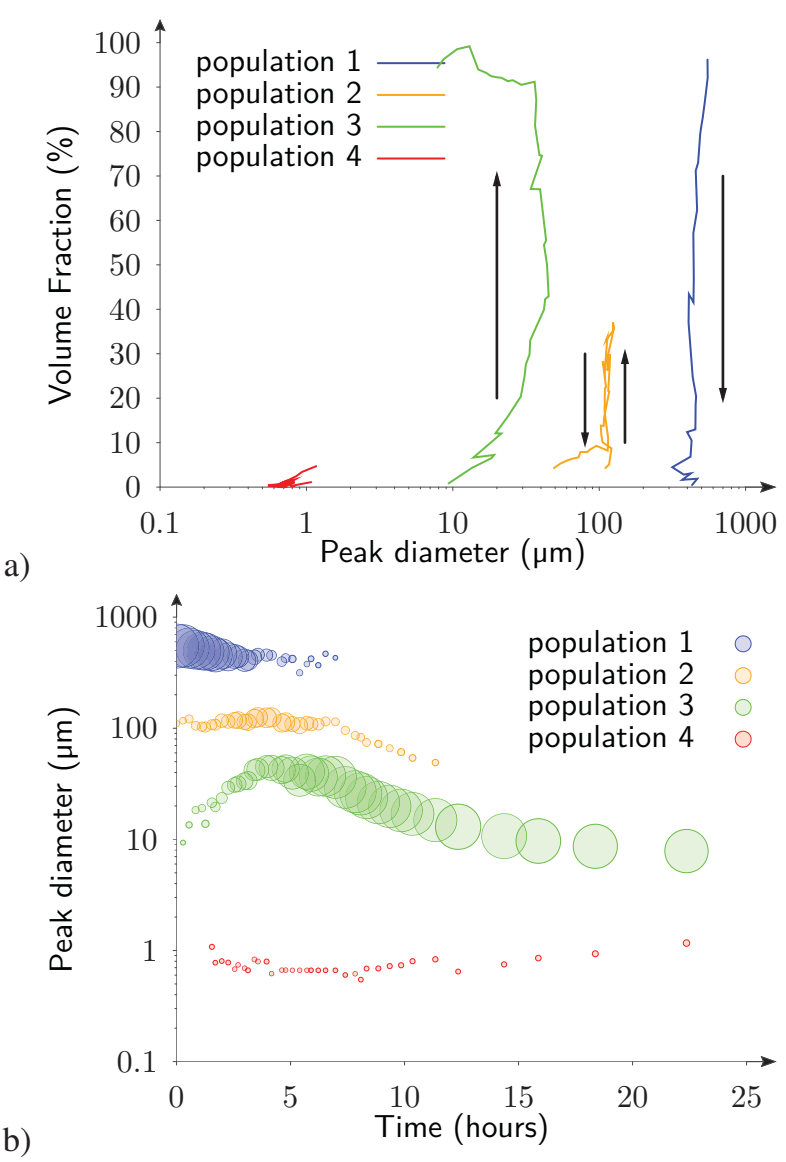

Figure 4. (a) Volume fraction as a function of the peak diameter (the arrows indicate the direction of time) (b) Peaks diameter as a function of time (the size of circles indicate the volume fraction)

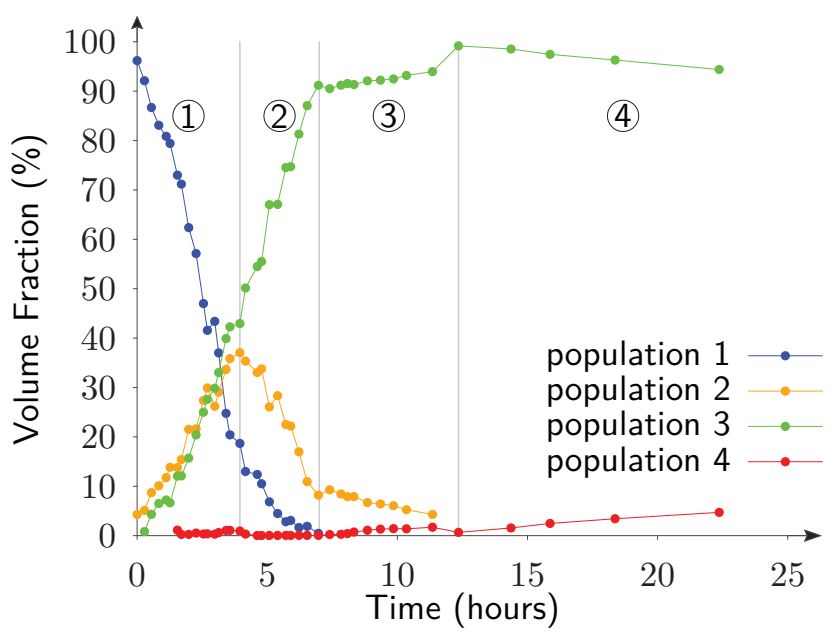

Figure 5. PSDs volume fraction as a function of time. Four different phases are identified.

However, since the population modes are not constant, it is difficult to compute an energy balance based on the variation of the volume fractions. In order to study the evolution of the specific surface, which reflects the energy used to create new surfaces, we assume that the particles 
are spherical and that their diameters corresponds to the size of the classes.

The Specific Surface Density (SSD) in the class $i$ and for the population $p$ is thus:

$$
S_{p}^{i}=\frac{6 \mathcal{V}_{p}\left(s^{i} ; \mathcal{X}_{p}\right)}{s^{i}}
$$

and the total specific surface per unit volume for population $p$ is:

$$
S_{p}=\sum_{i} S_{p}^{i}
$$

In Figure 6 the total SSD of the powder is increasing at constant rate during the first two phases. Then, this rate seems to saturate denoting a drop in the ability of grinding of the product. The energy supplied by the mill can be insufficient or the modification of the particles' microstructure with the decrease of the size [11] may change the response to impact or shearing solicitations.

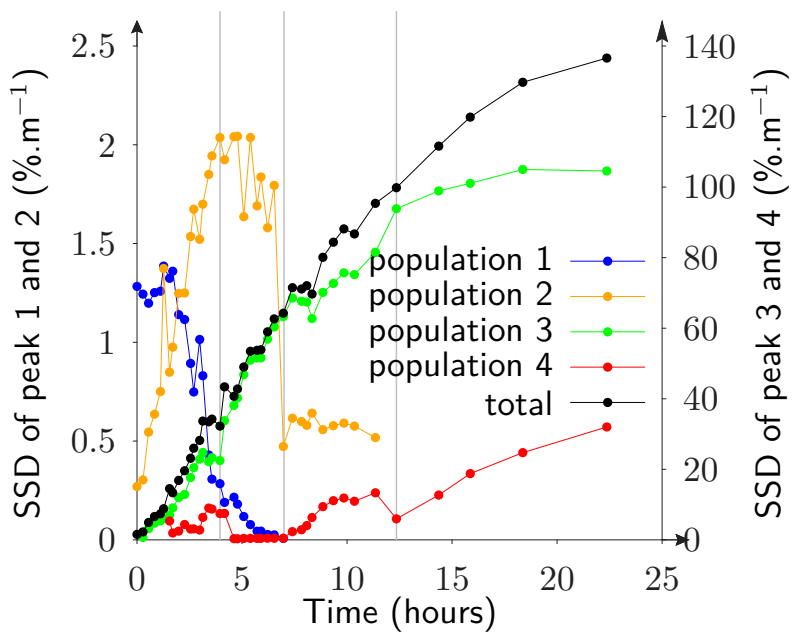

Figure 6. Specific surface density of the PSD and the different population as a function of time

It is interesting to note that the SSD curves do not reflect exactly the same phases as depicted in Figure 5.

The SSD of the population 2 exhibits instabilities during the second phase as its surface doesn't decrease regularly. This is due to instabilities in the values of $a_{p}$ and $b_{p}$ which can take very small values leading to a drastic increase of the SSD. Up to 1.7 hours, population 1 tends to stay constant showing that the increase in surface balances the loss of mass. The same behavior is visible for population 3 after 12 hours as its SSD is still increasing while the volume fraction declines. We note also that, because populations 1 and 2 represent large sizes, their contribution to the total SSD is small compared to the ones of populations 4 and 3.

\section{Concluding remarks and perspectives}

In this paper we proposed a methodology for the analysis of the grading curves of powders obtained by milling. The method is based on a deconvolution procedure which helps to identify characteristic sizes, corresponding to subPSDs. One important result was to be able to follow the evolution of the modes of the different populations in the course of grinding. This method can help to determine energy balance between the different populations, and to better understand how the grinding energy is employed at the particle scale for the creation of surface.

Regarding the jar mill process used in this work, we measured in details the evolution of the particle size distribution of wheat straw by taking 41 samples at different milling time. This process seems well adapted until the size of cellular scale corresponding to $20 \mu \mathrm{m}$ (population 3 ). This size allows the separation of different chemical components interesting for applications in biofuel or biomaterials. Note however that a finer milling is of great interest for many applications as it increases the reactivity and the accessibility of specific molecules.

In future work we would like to study the effect of the fracture mechanisms at the scale of particles on the evolution of the populations. One possibility is to associate to the grading curves some chemical information obtained by X-ray photoelectron spectroscopy or Laserinduced breakdown spectroscopy [12] as a function of the particle size. Finally, an industrial outlook is to compare the efficiency of different milling technologies for several types of biomass.

\section{Acknowledgments}

We thanks Céline Contesto and Cécile Barron for helpful discussions.

\section{References}

[1] A. Barakat, H. de Vries, X. Rouau, Bioresour. Technol., 134, 362-373 (2013)

[2] G. Ghizzi D. Silva, S. Guilbert, X. Rouau, Powder Technol, 208, 2 266-270 (2011)

[3] G. Ghizzi D. Silva, Fractionnement par voie sèche de la biomasse lignocellulosique : broyage poussé de la paille de blé et effets sur ses bioconversions, (2011)

[4] D.L. Black, M.Q. McQuay, M.P. Bonin, Prog. Energ. Combust. 22(3), 267-306 (1996)

[5] C. Voivret, F. Radjai, J.-Y. Delenne, M. S. El Youssoufi, Physical Review E 76, (2007)

[6] K. Levenberg, Q. Appl. Math. 2, 164-168 (1944)

[7] D. Marquardt, SIAM J. Appl. Math. 11(2), 431-441 (1963)

[8] F. Devernay, $\mathrm{C} / \mathrm{C}++\quad$ Minpack,http://devernay.free.fr/hacks/cminpack/ (2007)

[9] L. M. Popplewell, M. Peleg, Powder Technology, 2130 (1992)

[10] C. Voivret, F. Radjai, J.-Y. Delenne, M. S. El Youssoufi, Phys. Rev. Lett. 102, 178001 (2009)

[11] Yang, Y., Ji, G., Xiao, W. et al. Cellulose, 21, 5, 3257-3268 (2014)

[12] Milena R. Martelli, F. Brygo, A. Sadoudi, P. Delaporte, and C. Barron, J. Agr. Food Chem., 58,12, 71267134 (2010) 